\title{
Maternal serum triglyceride at midpregnancy and newborn weight in nondiabetic and normal BMI women
}

\author{
Leila Sekhavat, Fatemah Zare, Sedighah Akhavan Karbasi ${ }^{1}$ \\ Department of Obstetrics \& Gynecology and pediatericl, Shaheed Sadoughi hospital, \\ Shaheed Sadoughi University of Medical Sciences and Health Services, Yazd, Iran
}

\begin{abstract}
Aim: To determine whether elevated midpregnancy (24 -28 Gestational weeks) maternal serum lipid levels predict the risk of macrosomia in non-diabetic women.

Methods: Nondiabetic pregnant women (who had negative diabetic screens) were enrolled. Fasting serum triglyceride, and total cholesterol levels were measured at 24-28 weeks gestation. We tested the association between maternal variables and birth weight by univariable analysis. We also used multiple logistic regression analysis to determine whether maternal hyperlipidemia is a risk factor for having an LGA infant.

Results: We enrolled 190 subjects. Among measured maternal lipids, only triglyceride levels correlated with birth weight in univariable analysis $\left(r^{2}=0.40, P=.000\right)$. Logistic regression analysis showed that fasting maternal hypertriglyceridemia was the significant predictor of macrosomia, independent of maternal weight gain and fasting plasma glucose levels. (Odds ratio $11.2 ; 95 \%$ confidence interval 1.5, 31.2; $P=.01$ ).

Conclusion: In nondiabetic women, fasting triglyceride levels at midpregnancy correlated positively with newborn weight at term.
\end{abstract}

Keywords: Birth weight, hypertriglyceridemia, nondiabeic pregnant, normal BMI

\section{Introduction}

Fetal macrosomia, arbitrarily defined as a birth weight of more than $4250 \mathrm{gm}^{1}$. Fetal macrosomia is one of the major complications of diabetic pregnancy. ${ }^{2}$ However strict glycemic control sometimes failed to prevent macrosomia. ${ }^{3}$ It was reported that the risk of having macrosomia was reduced if intensive metabolic control was begun before but not after 32 weeks' gestation., ${ }^{4,5}$ Those reports suggested that macrosomia is associated with maternal metabolic condition at a certain gestational age.

In previous studies, significantly higher incidence of macrosomic infants was observed in women with normal diabetic screening. ${ }^{6,7}$ These findings suggested that fetal growth is determined largely by maternal factors, not only plasma glucose levels but also other fuels, such as lipids and amino acids, especially in nondiabetic women. ${ }^{8}$ Maternal serum lipid levels increase during mid to late gestation, which is believed to be beneficial to mother and fetus in terms of lactation and nutrition. ${ }^{9}$ Pregnancy is associated with increases in plasma total cholesterol and triglycerides. ${ }^{10}$ Maternal hyperlipidemia during pregnancy is associated with increased lipogenesis during the first two thirds of gestation. Maternal fat accumulation peaks in mid gestation and decreases in late gestation, whereas maternal serum lipid levels increase in mid to late pregnancy. ${ }^{11}$ It is considered a maternal adaptation to maintain stable fuel distribution to the fetus. In animal studies, shortage or excess of lipids in maternal circulation affected fetal growth and morbidity. ${ }^{12}$ However, the role of maternal hyperlipidemia in fetal growth is still not known, but

Correspondence

Leila Sekhavat, $M D$

Department of Obstetrics \& Gynecology, Shahid Sedughi Hospital, Shahid Sedughi University of Medical Sciences and Health Services, Yazd, Iran. Email:sekhavat@ssu.ac.ir

Fax No: 00983518224100, Tel: 00983518224001; Cell No. 00981525637 
although treatment with hypocholesterolemic drugs in pregnant rats has been shown to impair fetal growth. ${ }^{13}$ A recent study reported that postprandial triglyceride at midpregnancy was significantly associated with birth weight, and fetal macrosomia related strongly with impaired serum lipoprotein concentrations and composition. ${ }^{14}$ However, it is not known whether midpregnancy maternal fasting triglyceride levels are associated with birth weight, independent of maternal glucose levels and weight gain, and it has not been documented conclusively whether elevated triglyceride levels are associated with the risk of fetal macrosomia.

We studied nondiabetic women with negative diabetic screen. Our objective was to determine whether maternal serum lipid levels, including triglyceride, and total cholesterol, at $24-28$ gestational weeks are associated with newborn weight at term, and therefore, associated with a risk of developing macrosomia.

\section{Methods}

In a prospective, clinical trial study, we enrolled190 Iranian pregnant women who had normal BMI (prepregnancy BMI $=19-25$ ) and negative diabetic screen test results at 24-28weeks' gestation at Shahid Sadughi University Hospital within 2 years (2001 - 2003). The excluded criteria were: 1-Women with pregestational or gestational diabetes mellitus to eliminate therapeutic biases in the association between maternal metabolic variables and fetal growth. 2- Women with hypertensive disorder and preeclampsia. 3- Women with thyroid disorder, lupus, and antiphospholipid syndrome, because those conditions are associated with fetal growth restriction due to placental insufficiency rather than metabolic factors. 4- Subjects who delivered before 37 weeks' gestation, cases of fetal congenital malformations and cases of multifetal gestation.

At 24-28 gestational weeks, after an overnight fast, fasting blood samples were drawn to measure plasma glucose, serum triglyceride, and total cholesterol levels. Then we use of the 50-g oral glucose challenge test for screening. A 50-g oral glucose load is given without regard to the time elapsed since the last meal. Plasma glucose was measured by the oxidase method. Serum triglyceride and total cholesterol were measured using enzymatic methods. Prepregnant body mass index (BMI) was calculated from prepregnant weight and height reported by subjects and midwife's reports. Maternal weight gain during pregnancy was defined as an increase in weight from prepregnant weight to weight at the last visit. Gestational age was estimated by last menstrual period and confirmed by early fetal ultrasonographic measurements in all subjects. The criteria included FBS $>90 \mathrm{mg} / \mathrm{dL}$, and plasma or serum glucose one hour after $50-\mathrm{g}$ oral glucose $>130 \mathrm{mg} / \mathrm{dL}$ at $24-28$ gestational age, were given a diagnosis of GDM and excluded from the study[15].

All subjects were followed up until delivery. Neonatal birth weight more than $4250 \mathrm{~g}$ was defined macrosomia. ${ }^{1}$ We used univariable linear regression analysis to evaluate the association between each lipid concentration and newborn weight at term. We also used multiple logistic regression analysis to test the hypothesis that maternal hyperlipidemia in midpregnancy is a risk factor for LGA. Maternal hyperlipidemia was defined as a value higher than the 75 th percentile value of each lipid concentration. In the model, we used maternal fasting plasma glucose concentration and weight gain during pregnancy as confounding variables. The $\chi^{2}$ test was used to compare the incidence of macrosomic infants between women with and without hyperlipidemia. We used SPSS version 15.0 for statistical analysis, and $P<.05$ was defined as significant.

\section{Results}

190 women were studied. Maternal clinical and metabolic characteristics and the correlation coefficient between each variable and newborn weight are summarized in Table 1 . As well as maternal fasting serum triglyceride levels and maternal weight gain during pregnancy correlated significantly with newborn weight at term in univariable analysis. Fasting plasma glucose levels correlated positively but not significantly with newborn weight. Birth weight did not correlate significantly with the fasting total cholesterol levels. However, as expected, fasting triglyceride levels correlated significantly with maternal weight gain during pregnancy $\left(r^{2}=0.20, P=.04\right)$.

Hypertriglyceridemia was defined as more than the 75th percentile value $(259 \mathrm{mg} / \mathrm{dL})$ of all subjects. The incidence of macrosomia was significantly higher in mothers with hypertriglyceridemia (10 of $47,23.4 \%$ ) than in mothers who had normal triglyceride levels (8 of 143, $0.06 \%)(P=.000)$. We applied a logistic regression model with confounding variables, including fasting plasma glucose levels and weight gain during pregnancy, to test whether maternal hypertriglyceridemia predicts risk of having a macrosomic infant at term, independent of fasting plasma glucose and maternal weight gain. In the model, hypertriglyceridemia at 24-18 weeks' gestation was a significant predictor of having a macrosomic infant at term (odds ratio 11.2; $95 \%$ confidence interval 1.5, 31.2; $P=.01$ ), independent of maternal glucose levels and weight gain (Table2). 
Table I. Clinical characteristics, glucose, lipid levels and birth weight

\begin{tabular}{lcccc}
\hline Clinical variable & Mean \pm SD & Range & Correlation coefficient $(r)^{*}$ & $P$ \\
\hline Maternal age (y) & $27.5 \pm 5$ & $20-41$ & 0.11 & .10 \\
Gestational age at delivery (wks) & $38 . \pm .8$ & $37-41$ & -0.03 & 0.64 \\
Maternal weight gain during pregnancy (kg) & $11.4 \pm 2.4$ & $5-19$ & 0.35 & .002 \\
Birth weight (g) & $3520 \pm 521$ & $2250-4650$ & & .030 \\
Fasting plasma glucose (mg/dL) & $80.2 \pm 5.4$ & $65-90$ & 0.22 & .000 \\
Fasting serum triglyceride (mg/dL) & $213.9 \pm 77.7$ & $86-476$ & 0.40 & .18 \\
Fasting serum total cholesterol (mg/dL) & $214.3 \pm 92$ & $135-425$ & 0.08 & \\
\hline
\end{tabular}

* The association between newborn birth weight and each clinical variable by univariable analysis

Table 2. Risk of macrosomic infants: Multiple logistic regression model

\begin{tabular}{lccc}
\hline Predictive variable & OR & $95 \% \mathrm{Cl}$ & $P$ \\
\hline Hypertriglyceridemia* & 11.2 & $1.5,31.2$ & .01 \\
Fasting plasma glucose (-mg/dL) & 1.04 & $0.12,1.47$ & .35 \\
Maternal weight gain $(\mathrm{kg})$ & 1.17 & $2.02,4.14$ & .62 \\
\hline
\end{tabular}

* Hypertriglyceridemia is defined as having fasting serum triglyceride levels above the 75 th percentile level (>259 $\mathrm{mg} / \mathrm{dL})$, $O R=$ odds ratio, $I=$ confidence interval

\section{Discussion}

In this study, we found that maternal fasting serum triglyceride levels at 24-28 weeks' gestation were significantly and positively associated with newborn weight at term. The association was independent of weight gain during pregnancy or midpregnancy plasma glucose levels (either fasting or postprandial). The study by Merzouk et $\mathrm{al}^{14}$ in which similar eligibility criteria were used, found that triglyceride level after 50 -g oral glucose screening load at 24-28 weeks' gestation was significantly associated with adjusted newborn weight, independent of maternal obesity. The correlation coefficient was higher in our study $(r=0.22$, $P<.01)$ than in Merzouk's report $(r=0.13, P<.05)$. Difference in maternal conditions, the fasting or the postprandial state, might have caused the variation in the studies. Although some studies found an association between maternal free fatty acid concentration and neonatal birth weight.${ }^{12}$ We did not find any relation of either total cholesterol to newborn weight.

We also showed subjects who had fasting serum triglyceride levels above the 75th percentile at 24-28 weeks' gestation were at significant risk of having macrosomic infants at term, independent of maternal weight gain during pregnancy, and plasma glucose levels. Our findings regarding the effect of midpregnancy maternal hypertriglyceridemia on fetal overgrowth might be limited to non-diabetic women. Recent reports suggest that women with negative results of diabetic screen might be at risk of macrosomia.${ }^{6,7}$ In addition, Merzouk et al ${ }^{14}$ who found a positive association between serum triglyceride levels after 50 -g oral glucose load and adjusted newborn weight in women whose diabetic screen results were negative. Therefore, maternal triglyceride measurements in midpregnancy might have important clinical value to predict fetal overgrowth at term in nondiabetic pregnancies.

Another interesting finding in our study was the relationship between maternal plasma glucose levels and newborn weight. In non-diabetic pregnancies, it is still controversial whether midpregnancy maternal fasting or postprandial plasma glucose level is an independent predictor of newborn weight at term. In the current study, newborn weight at term correlated significantly with fasting plasma glucose but not with postprandial levels at 24-28 weeks' gestation. However, in the multiple logistic regression model that included maternal triglyceride and maternal weight gain during pregnancy as covariates, the association between fasting glucose level and birth weight remains statistically significant $(P=.02)$. This result is controversy with another recent study in which there was significant effect of plasma glucose values on macrosomia in screening-positive and GTT-negative subjects. ${ }^{16}$ Although it might be a result of a lack of statistical power because of our small sample size, it seemed that maternal serum triglyceride level had a more important effect on fetal growth than maternal plasma glucose in non-diabetic women. 
The positive association between maternal triglyceride level and newborn weight is confusing, because maternal serum triglyceride does not appear to cross the placenta. ${ }^{17}$ With respect to the physiologic mechanism, it is hypothesized that hydrolysis of maternal triglyceride by placental lipoprotein lipase to free fatty acids that cross the placenta is increased. ${ }^{17}$ Enhanced insulin resistance during late pregnancy would explain the association between maternal triglyceride level and fetal growth. Hypertriglyceridemia is one of the major characteristics of insulin resistance syndrome in non-pregnant adults. ${ }^{18}$ Also in pregnancy, hyperlipidemia including hypertriglyceridemia was found in women with gestational diabetes which is associated with both fetal macrosomia and decreased insulin sensitivity during pregnancy compared with normal pregnancy. ${ }^{18-20}$ Conversely, some authors reported that maternal insulin sensitivity was negatively associated with infantile birth weight. ${ }^{21,22}$ We speculate that maternal hypertriglyceridemia in midpregnancy reflects increased insulin resistance, and consequently, is associated with newborn birth weight.

\section{Conclusion}

In nondiabetic with normal prepregnancy BMI women, fasting triglyceride levels at 24-28 weeks' gestation correlated positively with newborn weight at term; Therefore pregnant benefit with controlling their diet (low lipid diet)

\section{References}

1. Zamorski MarkA, Wendy S. Management of suspected fetal macrosomia, American Family Physician, 2001, 63:15.

2. de Valk HW, van Nieuwaal NH, Visser GH. Pregnancy outcome in type 2 diabetes mellitus: a retrospective analysis from the Netherlands. Rev Diabetes Stud, 2006, 3:134-42.

3. Langer O, Yogev Y, Most O, Xenakis EM. Gestational diabetes: the consequences of not treating. Am J Obstet Gynecol 2005, 192: 989-92.

4. Lin CC, River J, River P. Good diabetic control early in pregnancy and favorable fetal outcome. Obstet Gynecol, 1986, 67:51-6.

5. Eleni NE, Dimitrios N, Kiortsis ET. Lipid profile, glucose homeostasis, blood pressure and
Obesity-Anthropometric Markers in macrosomic offspring of nondiabetic Mothers. Diabetes Care 2006; 29: 1197-1201.

6. Orskou J, Henriksen TB, Kesmodel U. Maternal characteristics and lifestyle factors and the risk of delivering high birth weight infants. Obstet Gynecol 2003:102-115.

7. Okun N, Verma A, Mitchell BF. Relative importance of maternal constitutional factors and glucose intolerance of pregnancy in the development of newborn macrosomia. J Matern Fetal Med 1997; 6: 285-90.

8. Sørensen HT, Sabroe S, Rothman KJ, Gillman M, Fischer P, Sørensen TI. Relation between weight and length at birth and body mass index in young adulthood, cohort study. BMJ 1997 Nov 1; 315(7116):1137.

9. Herrera E, Munilla MA. Maternal lipid metabolism and its implications for fetal growth. In: Battaglia FC, ed. Placental function and fetal nutrition. Nestlé Nutrition Workshop Series 1997; 39: 169-82.

10. Clausen T, Burski T.K, Oyen N, Godang K, Bollerslev. Maternal anthropometric and metabolic factors in the first half of pregnancy and risk of neonatal macrosomia in term pregnancies. A prospective study. Eur $J$ Endocrinol 2005; 153: 887-894.

11. Knopp. RH, Bonet. B, Zhu. X. Lipid metabolism in pregnancy. In: Cowett RM, ed. Principles of perinatal-neonatal metabolism; 1998; $2^{\text {nd }}$ edn: 221-58.

12. McGladdery SH, Frohlich JJ. Lipoprotein lipase and apoE polymorphisms: relationship to hypertriglyceridemia during pregnancy. $J$ Lipid Res 2001 Nov; 42(11):1905-12.

13. Vtr M. Risk factors associated with high birthweight deliveries. Ceska Gyneko, 2005; 70: 347-54.

14. Merzouk H, Bouchenak M, Loukidi B. Fetal macrosomia related to maternal poorly controlled type 1 diabetes strongly impairs serum lipoprotein concentrations and composition. Clin Pathol 2000; 53:917-923. 
15. ACOG Practice Bulletin. Clinical management guidelines for obstetrician-gynecologists 2001, Sept 30 .

16. Michio K, Satoshi O, Ichiro Y. Maternal Serum Triglyceride at 24-32 Weeks' Gestation and Newborn Weight in Nondiabetic Women with Positive Diabetic Screens. Obstetrics and Gynecology 2001; 97: 776-780.

17. Mantzoros. CS, Flier. JS, Mazzaferi. E. Insulin resistance, the clinical spectrum. Advances in endocrinology and metabolism 1995; 6:193232.

18. Sevillano J, López-Pérez IC, Herrera E, Del Pilar Ramos M, Bocos C. Englitazone administration to late pregnant rats produces delayed body growth and insulin resistance in their fetuses and neonates. Biochem J 2005 Aug 1;389(Pt 3):913-8.
19 Meyers-Seifer CH, Vohr BR. Lipid levels in former gestational diabetic mothers. Diabetes Care 1996; 19: $1351-6$.

20. Catalano PM, Drago NM, Amini SB. Maternal, carbohydrate metabolism and its relationship to fetal growth and body composition. Am J Obstet Gynecol 1995; 172: 1464-70.

21. Caruzo A, Paradisi G, Ferrazzani. S. Effect of maternal carbohydrate metabolism on fetal growth. Obstet Gynecol 1998; 92: 8-12.

22. Di Cianni G, Miccoli R, Volpe L, Lencioni C, Ghio A, Giovannitti MG, Cuccuru I, Pellegrini G, Chatzianagnostou K, Boldrini A, Del Prato S. Maternal triglyceride levels and newborn weight in pregnant women with normal glucose tolerance. Diabet Med. 2005 Jan; 22(1):21-5. 